\title{
On the Crystallinity and Durability of ZDDP Tribofilm
}

\author{
Mao Ueda ${ }^{1} \cdot$ Amir Kadiric $^{1} \cdot$ Hugh Spikes $^{1}$ \\ Received: 24 April 2019 / Accepted: 17 October 2019 / Published online: 30 October 2019 \\ (C) The Author(s) 2019
}

\begin{abstract}
The current trend for using lower-viscosity lubricants with the aim of improving fuel economy of mechanical systems means that machine components are required to operate for longer periods in thin oil film, mixed lubrication conditions, where the risk of surface damage is increased. Consequently, the performance and durability of the tribofilms formed by antiwear additives, and in particular zinc dialkyldithiophosphate (ZDDP), the main antiwear oil additive used in engine oils, has become an increasingly important issue. In this paper, it is confirmed that ZDDP tribofilms are initially relatively easily removed by rubbing but that they become more durable during prolonged rubbing. FIB-TEM analyses at different stages of tribofilm formation show that during the early stages of rubbing only the tribofilm close to the steel substrate is nanocrystalline, while the outer region is amorphous and easily removed. However, after prolonged rubbing all regions of the tribofilm become nanocrystalline and able to withstand rubbing in base oil without being removed. XPS analysis shows that after extended rubbing the outermost polyphosphate structures change from longer-chain structures such as metaphosphate and polyphosphate to shorter-chain structures including orthophosphate. This depolymerization of ZDDP tribofilm from long- to short-chain phosphate and consequent nanocrystallization are driven by heat and shear stress. EDX analysis shows that this conversion is promoted by diffusion of Fe cation into the bulk of the tribofilm. The finding that ZDDP tribofilms evolve during rubbing from a weaker amorphous structure to a more durable nanocrystalline one has important implications in terms of the behaviour of ZDDPs at low concentrations, on non-metallic surfaces and at very high contact pressures, as well as for the development of ZDDP tribofilm, friction and wear models.
\end{abstract}

Keywords ZDDP $\cdot$ Crystallinity $\cdot$ Amorphous $\cdot$ Depolymerization $\cdot$ Durability

\section{Introduction}

In recent years, the role of ZDDP as an antiwear additive in engine lubricants has become increasingly important because of the introduction of very low-viscosity oils in order to reduce hydrodynamic friction and thus increase engine efficiency. Such low-viscosity oils can cause the engine components to operate for long periods in thin film, mixed lubrication conditions, where wear of rubbing surfaces may occur. Therefore, the ability of ZDDP tribofilms to survive over long rubbing times, called ZDDP tribofilm durability, is an important characteristic.

One way to assess the durability of a ZDDP tribofilm is to monitor changes in ZDDP tribofilm thickness after the

Mao Ueda

m.ueda18@imperial.ac.uk

1 Imperial College London, Tribology Group, London SW7 2AZ, UK replacement of a ZDDP-containing oil by a ZDDP-free oil [1-3]. Fujita et al. [1] measured the growth of a thick ZDDP tribofilm in rolling-sliding contact using optical interferometry and then the effect on this pre-formed tribofilm of replacing the ZDDP solution by base oil. They found that the thickness of the ZDDP tribofilm remained almost unchanged when rubbed in base oil, indicating that it was very durable in rolling-sliding conditions. If, however, the ZDDP solution was replaced by a solution of a succinimide dispersant, about half to two-thirds of the pre-formed tribofilm thickness was rapidly removed $[1,2]$. They suggested that some of the tribofilm was susceptible to extraction of stabilizing $\mathrm{Zn}$ cations by the aminic dispersant. It thus appeared that, in the absence of specific chemical effects, ZDDP tribofilms were extremely durable.

This picture has, however, recently become more complicated since Parsaeian et al. [3] have shown that the durability of a ZDDP tribofilm appears to depend on how long it has been rubbed. Like Fujita et al., they found that if ZDDP 
solution was replaced by base oil after prolonged rubbing, the tribofilm was not significantly removed during further rubbing and was thus durable. If, however, the ZDDP solution was replaced by base oil in the early stages of a test, and rubbing continued, some of the tribofilm was removed. Partial tribofilm removal was also observed when the applied load or temperature was increased in the early stages of a test. The authors also showed that, while durable tribofilm was composed of shorter polyphosphates, vulnerable tribofilm was composed of longer polyphosphates. The chain length of phosphates in ZDDP tribofilms can be estimated from the ratio of bonding oxygen, BO (P-O-P), to non-bonding oxygen, $\mathrm{NBO}(\mathrm{P}=\mathrm{O}$ and $\mathrm{P}-\mathrm{O}-\mathrm{M})$, intensity, the $\mathrm{BO} / \mathrm{NBO}$ ratio [4-7]. It should be noted that many studies have shown that the chain length of zinc polyphosphate reduces with rubbing time [3, 6, 8-10] though a few have proposed precisely the opposite [11, 12]. Parsaeian et al.'s work [3] thus suggests that the strength/durability of ZDDP tribofilm may depend on its phosphate chain length and thus change with rubbing time. However, the reason why phosphate chain length might affect tribofilm durability is not clear.

One possibility is that the phosphate chain length may influence the structure of the ZDDP tribofilm and in particular its crystallinity. It is well known that the extent of crystallinity can affect the properties of structures and that nanocrystalline materials can have very high strength relative to amorphous ones [13]. Most previous research has found or assumed that ZDDP tribofilms consist primarily of amorphous and glassy zinc phosphates. However, using high-resolution FIB-TEM it has recently been shown that ZDDP tribofilms may have both nanocrystalline and amorphous structures [14].

Crobu et al. [5] determined the structures of synthesized metaphosphate and orthophosphate using XRD analysis. While pure metaphosphate showed amorphous peak patterns, pure orthophosphate showed crystalline XRD patterns. Thus, the reduction in tribofilm phosphate chain length may be accompanied by the formation of a crystalline structure.

Based on the above, the aim of this paper is to understand the evolution of ZDDP tribofilm crystallinity during its formation and the effect of this on its durability. Reaction mechanisms of tribofilm crystallinity formation are also suggested. Tribofilm durability is investigated at different rubbing times using MTM-SLIM, with parallel observation of tribofilm crystallinity using the FIB-TEM method. Also, XPS is used to understand the relationship between zinc phosphate structures and tribofilm crystallinity.

\section{Test Lubricants}

Solutions of two ZDDPs in a PAO base oil were studied. The base oil was PAO 4 having viscosity $18.5 \mathrm{~mm}^{2} / \mathrm{s}$ at $40{ }^{\circ} \mathrm{C}$ and $4.1 \mathrm{~mm}^{2} / \mathrm{s}$ at $100{ }^{\circ} \mathrm{C}$. One ZDDP (ZDDP1) was a primary $\mathrm{C} 8$ and the other (ZDDP2) a secondary C6. Both were used at a concentration of $0.08 \mathrm{wt} \% \mathrm{P}$.

\section{Test Methods}

\subsection{MTM Test Rig}

A mini-traction machine (MTM) ball on disc tribometer with a spacer layer imaging attachment (SLIM) was employed to observe ZDDP tribofilm growth. The rig is shown in Fig. 1. A steel ball is loaded against a steel disc which is immersed in lubricant. The ball and the disc are driven by separate electric motors at specified rolling/sliding conditions. To capture SLIM images, the rubbing test is paused and the ball is raised and loaded against a glass flat. The glass flat surface is coated with a semi-reflective chromium layer on top of which is a transparent silica spacer layer. Light waves reflected from the chromium layer and the ball surface undergo optical interference upon recombination, generating an interference image of the contact between the ball and the glass surface which is then captured by an RGB camera. Tribofilm thickness is calculated from this interferometry image using calibrated RGB values.

\subsection{Test Conditions and Procedures}

ZDDP tribofilm durability was evaluated by measuring the evolution of tribofilm thickness after the replacement of the ZDDP solution by PAO base oil. Most tests thus consisted of two stages, one in which the ball and disc were rubbed in ZDDP solution and the second in which the ball and disc, having pre-formed ZDDP tribofilms, were rubbed in PAO 4. Before this second stage, the ball and discs were removed from the test rig and rinsed in toluene to remove all ZDDP solution and then three times in

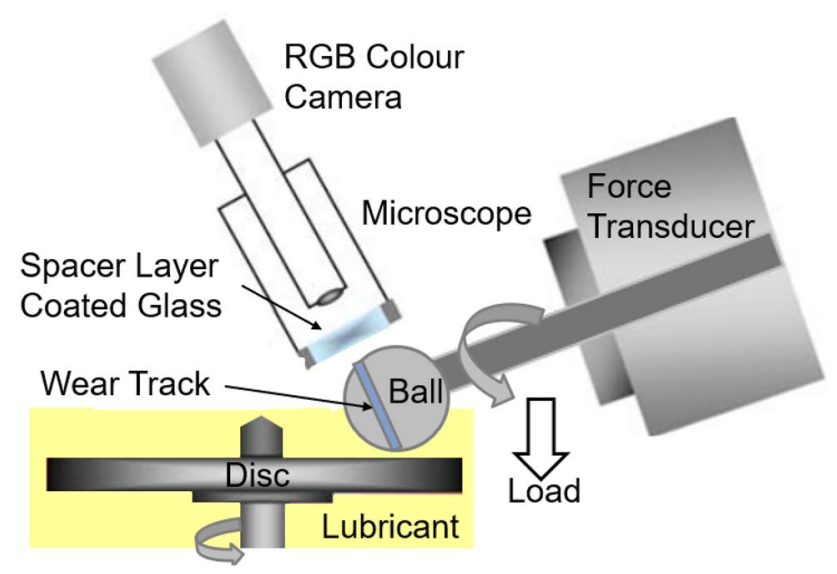

Fig. 1 Schematic image of the MTM-SLIM set-up 
PAO 4. They were then returned to the rig to be rubbed in PAO 4. Table 1 lists the invariant conditions used throughout all tests, while Table 2 lists the conditions that varied from test to test for all 16 tests carried out. Two test temperatures were studied, $60{ }^{\circ} \mathrm{C}$ and $100{ }^{\circ} \mathrm{C}$. At these two temperatures, the calculated lambda (ratio of EHD film thickness to composite surface roughness) was 0.04 and 0.02 , respectively.

To investigate the tribofilm properties, discs were cleaned by toluene in an ultrasonic bath for $10 \mathrm{~min}$ followed by the analysis (Tests 1, 3, 4, 6, 9, 12 and 14). Test 4 was stopped after 5-min rubbing in stage 2, and tribofilm on the disc was then analysed after cleaning to evaluate the property changes in tribofilm before and after the replacement of ZDDP solution with base oil. As indicated in Table 2 , the effect of temperature $\left(100{ }^{\circ} \mathrm{C}\right.$ and $\left.60{ }^{\circ} \mathrm{C}\right)$ and ZDDP alkyl structure (secondary type and primary type) was studied to understand the mechanism of tribofilm crystallinity formation.

Table 1 General MTM test conditions

\begin{tabular}{ll}
\hline Mean (entrainment $)$ speed; & $50(\mathrm{~mm} / \mathrm{s})$ \\
$\mathrm{U}=\left(\mathrm{U}_{\text {ball }}+\mathrm{U}_{\text {disc }}\right) / 2$ & \\
Slide-roll ratio $(\mathrm{SRR}) ;$ & $50 \%$ \\
$\mathrm{SRR}=100 \times\left(\mathrm{U}_{\text {disc }}-\mathrm{U}_{\text {ball }}\right) / \mathrm{U}$ & \\
Applied load & $31 \mathrm{~N}($ max Hertz pressure $0.95 \mathrm{GPa})$ \\
Ball & $19.05 \mathrm{~mm}$ diameter, AISI 52100, \\
& $855 \mathrm{HV}, \sim 0.012 \mu \mathrm{m} \mathrm{Ra}$ \\
Disc & AISI $52100,825 \mathrm{HV}, \sim 0.010 \mu \mathrm{m} \mathrm{Ra}$ \\
\hline
\end{tabular}

\subsection{Tribofilm Analysis}

\subsubsection{Tribofilm Crystallinity}

To investigate tribofilm crystallinity using transmission electron microscopy (TEM), lamellae of ZDDP tribofilm cross sections were extracted using a Helios 600 NanoLab Focused Ion Beam (FIB) instrument. Before this, the ZDDP tribofilms were coated with a 50-70-nm gold layer to protect them from damage during gallium ion milling [14]. Table 3 shows the conditions used in the FIB process. After the specimens were installed in a vacuum chamber, a Pt layer was deposited in an atmosphere of Ga. After bulk-out and U-cut milling processes using $\mathrm{Ga}$ ions, lamellae with tribofilm (width $10 \mu \mathrm{m}$, depth $7 \mu \mathrm{m}$, thickness $100 \mathrm{~nm}$ ) were lifted out using a tungsten omniprobe. These lamellae were then mounted on copper grids and thinned down and cleaned.

Table 3 Focused ion beam conditions for ZDDP tribofilm cross section preparation

\begin{tabular}{lll}
\hline Step & $\begin{array}{l}\text { Acceleration volt- } \\
\text { age }(\mathrm{kV})\end{array}$ & Beam current \\
\hline Pt deposition & 30 & $0.46 \mathrm{nA}$ \\
Bulk-out & 30 & $21 \mathrm{nA}$ \\
Cleaning of cross section & 30 & $6.5 \mathrm{nA}$ \\
U-cut & 30 & $6.5 \mathrm{nA}$ \\
Thinning & 30 & $0.46 \mathrm{nA}$ \\
Cleaning & 2 & $28 \mathrm{pA}$ \\
\hline
\end{tabular}

Table 2 Specific test conditions

\begin{tabular}{|c|c|c|c|c|c|c|c|c|}
\hline \multirow[t]{2}{*}{ Test \# } & \multicolumn{3}{|c|}{ Stage 1 conditions } & \multirow{2}{*}{$\begin{array}{l}\text { Tribofilm analysis } \\
\text { on the disc }\end{array}$} & \multicolumn{3}{|c|}{ Stage 2 conditions } & \multirow{2}{*}{$\begin{array}{l}\text { Tribofilm } \\
\text { analysis on the } \\
\text { disc }\end{array}$} \\
\hline & Oil & Temp $\left({ }^{\circ} \mathrm{C}\right)$ & $\begin{array}{l}\text { Rubbing time } \\
(\mathrm{min})\end{array}$ & & Oil & Temp $\left({ }^{\circ} \mathrm{C}\right)$ & $\begin{array}{l}\text { Rubbing time } \\
\text { (min.) }\end{array}$ & \\
\hline 1 & ZDDP2 & 100 & 30 & $\checkmark$ & No stage 2 & & & \\
\hline 2 & ZDDP2 & 100 & 30 & & PAO 4 & 100 & 210 & \\
\hline 3 & ZDDP2 & 100 & 60 & $\checkmark$ & No stage 2 & & & \\
\hline 4 & ZDDP2 & 100 & 60 & & $\mathrm{PAO} 4$ & 100 & 5 & $\checkmark$ \\
\hline 5 & ZDDP2 & 100 & 60 & & $\mathrm{PAO} 4$ & 100 & 180 & \\
\hline 6 & ZDDP2 & 100 & 180 & $\checkmark$ & No stage 2 & & & \\
\hline 7 & ZDDP2 & 100 & 180 & & $\mathrm{PAO} 4$ & 100 & 60 & \\
\hline 8 & ZDDP2 & 100 & 240 & & No stage 2 & & & \\
\hline 9 & ZDDP2 & 60 & 180 & $\checkmark$ & No stage 2 & & & \\
\hline 10 & ZDDP2 & 60 & 180 & & PAO 4 & 100 & 60 & \\
\hline 11 & ZDDP2 & 60 & 240 & & No stage 2 & & & \\
\hline 12 & ZDDP1 & 100 & 180 & $\checkmark$ & no stage 2 & & & \\
\hline 13 & ZDDP1 & 100 & 180 & & PAO 4 & 100 & 60 & \\
\hline 14 & ZDDP1 & 100 & 360 & $\checkmark$ & No stage 2 & & & \\
\hline 15 & ZDDP1 & 100 & 360 & & $\mathrm{PAO} 4$ & 100 & 60 & \\
\hline 16 & ZDDP1 & 100 & 420 & & No stage 2 & & & \\
\hline
\end{tabular}


To observe tribofilm crystallinity in these lamellae, TEM images were captured using a TEM (JEOL $2100 \mathrm{~F}$ ) with $200 \mathrm{kV}$ accelerating voltage. Fast Fourier transform (FFT) of the TEM images was also obtained. Scanning TEM (STEM) was then performed on JEOL-2100 F operated at $200 \mathrm{kV}$, equipped with an Oxford Instruments energy-dispersive $\mathrm{X}$-ray spectroscopy (EDS) detector. Elemental maps of tribofilm were collected on the samples tilted to $15^{\circ}$ using the Oxford Instruments X-ray System INCA.

\subsubsection{Tribofilm Chemical Characterization}

A Thermo Fisher K-Alpha spectrophotometer was used for X-ray photoelectron spectroscopy (XPS) to analyse the ZDDP tribofilms. A monochromatic Al-K $\alpha \mathrm{X}$-ray source $($ energy $=1486.6 \mathrm{eV}$ ) was employed. The X-ray source was operated at $6 \mathrm{~mA}$ emission current and $12 \mathrm{kV}$ anode bias. The calibration of the spectrometer was performed using $\mathrm{Au}$ (4f7/2), $\mathrm{Cu}(2 \mathrm{p} 3 / 2)$ and $\mathrm{Ag}(3 \mathrm{~d} 5 / 2)$ signals. The spot size was an elliptical shape with long axis of $200 \mu \mathrm{m}$ to make sure that analysis area was within the tribotrack. Samples were positioned at 0 degree emission angle. Base pressure in the main chamber of the spectrometer was $2 \times 10^{-9} \mathrm{mbar}$. A survey spectrum was acquired in a high-pass energy $(200 \mathrm{eV})$ scanned mode. A narrow scan, used to determine the relative atomic composition, as well as for determination of chemical information, was then acquired in a low-pass energy $(20 \mathrm{eV})$. The charging of the specimen was corrected by referring aliphatic carbon binding energy to $284.8 \mathrm{eV}$ (C 1s signal). The XPS analyses were carried out on the tribofilm surfaces on the discs without any sputtering. The $\mathrm{O} 1 \mathrm{~s}$ peak was curve-fitted to non-bridging bond (NBO) at $531.2 \pm 0.5 \mathrm{eV}$ and bridging oxygen (BO) at $532.6 \pm 0.5 \mathrm{eV}$ $[4,5]$.

\section{Results}

\subsection{Evolution of ZDDP Tribofilm Durability}

Figure 2 shows the effect of sliding time on ZDDP2 tribofilm durability at $100{ }^{\circ} \mathrm{C}$. In the case of rubbing for 240 min in ZDDP2 solution (Test 8), tribofilm thickness increased to $170 \mathrm{~nm}$ over the first $120 \mathrm{~min}$ of rubbing and subsequently remained stable until the end of the test. Tribofilm growth was relatively linear with time and negligible induction period observed. In the case of replacement of the ZDDP2 solution by PAO 4 after 180-min rubbing, none of the ZDDP tribofilm was removed (Test 7). However, in the case of replacement of the ZDDP2 solution by PAO 4 after only 30 - and 60-min rubbing, ca. 20- and 40-nm tribofilm, respectively, was removed from the ball after just 5-min rubbing in PAO (Tests 2 and 5). These results confirm that

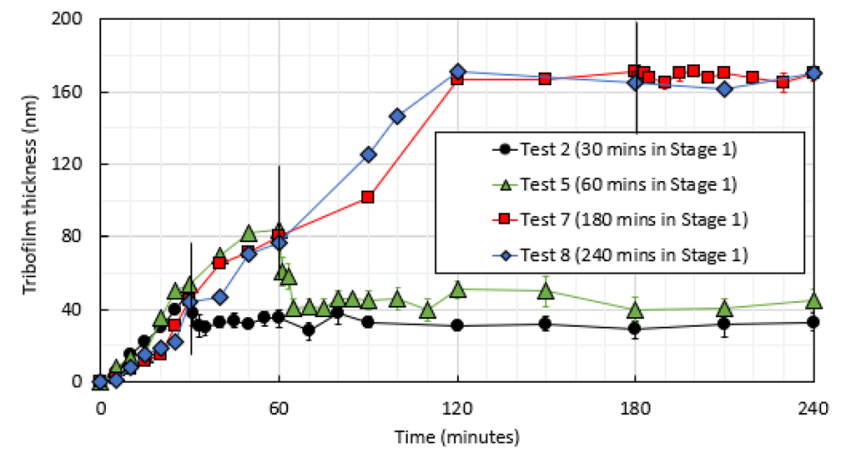

Fig. 2 ZDDP2 tribofilm evolution and its durability after different stage 1 rubbing times at $100{ }^{\circ} \mathrm{C}$ (Tests $2,5,7$ and 8)

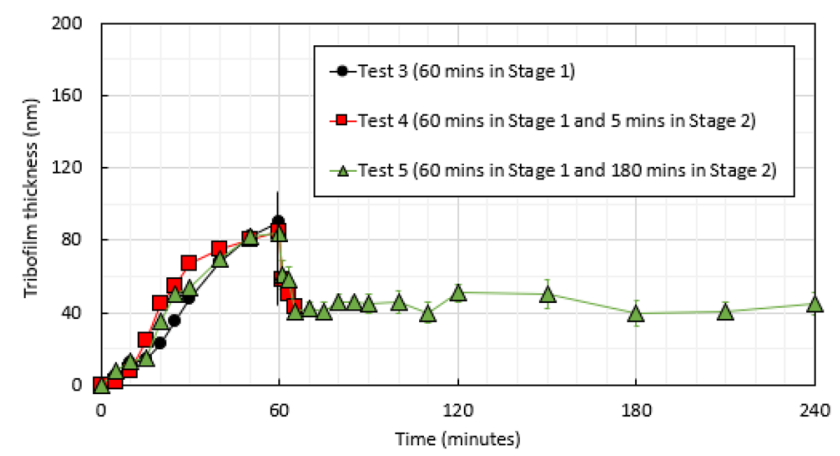

Fig. 3 ZDDP2 tribofilm evolution and its durability after $60 \mathrm{~min}$ in stage 1 and different stage 2 rubbing times at $100{ }^{\circ} \mathrm{C}$ (Tests 3,4 and 5)

ZDDP tribofilms become more durable with rubbing time. All tests were repeated twice, and the film thickness spread is shown in Figs. 2 and 3. To further illustrate repeatability, Fig. 3 shows the results for Tests 3, 4 and 5, all of which either replaced the test oil by PAO after 60 min or ended the test at this stage. It is evident that the rate and thickness of tribofilm formation are similar in each test.

\subsection{ZDDP Tribofilm Crystallinity}

\subsubsection{Tribofilm Crystallinity After Rubbing in ZDDP Solution}

Figure 4 shows cross-sectional TEM images of ZDDP tribofilms after 30-, 60- and 180-min rubbing in ZDDP2 solution at $100{ }^{\circ} \mathrm{C}$ (Tests 1,3 and 6). FFT taken from inside the black and white squares in the TEM images is also shown. In these TEM images, the black regions on the top of tribofilm represent the gold coating, while the grey regions on the bottom of tribofilm are the steel substrate. For the thick tribofilms formed after 60 and $180 \mathrm{~min}$, separate TEM images are shown of the lower side (closest to steel) and the 


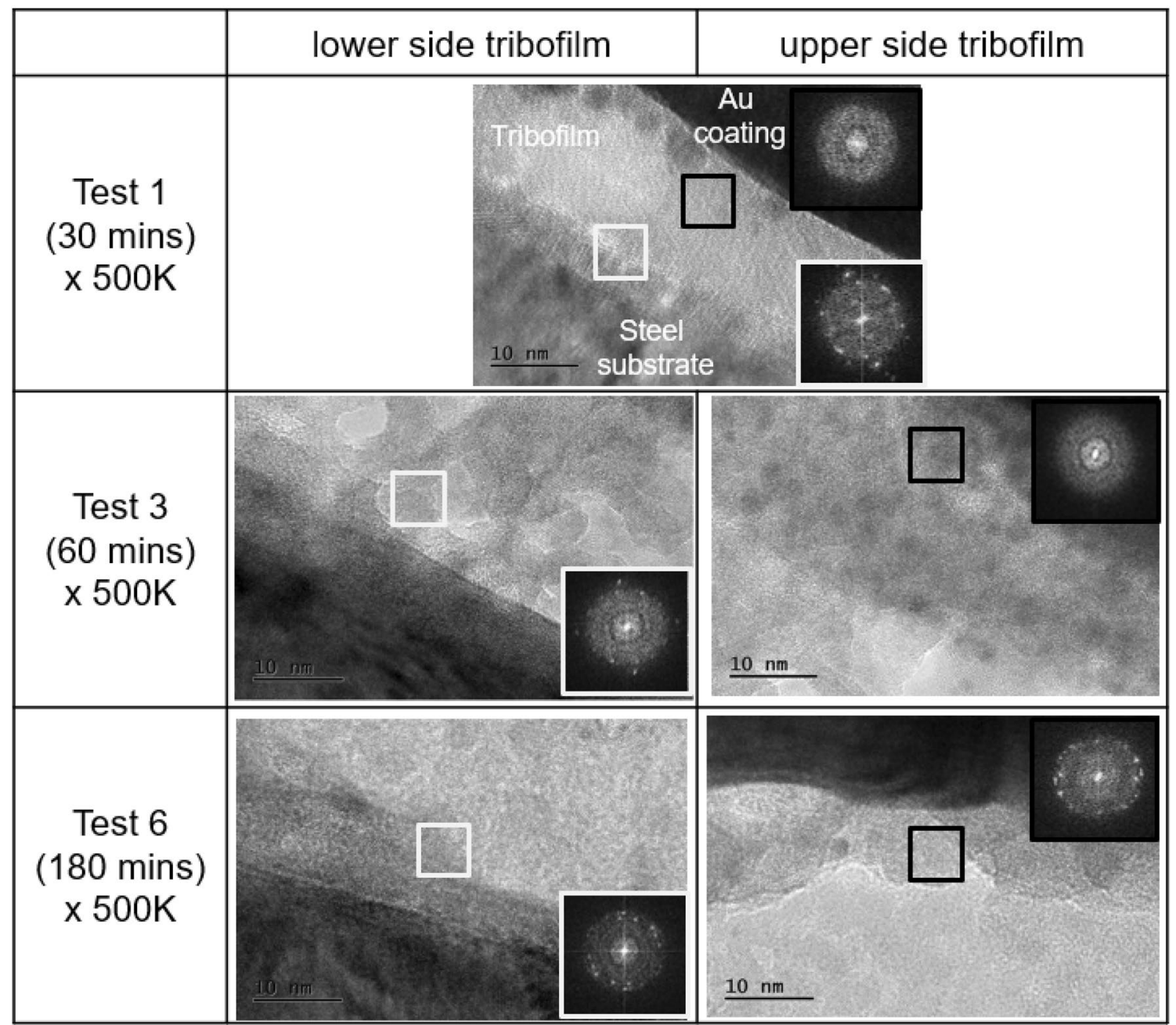

Fig. 4 TEM images and FFTs of the upper and lower side of tribofilms after 30-, 60- and 180-min rubbing in ZDDP2 solution (Tests 1, 3 and 6). Steel substrate is the dark grey region at the bottom of these images, while the upper black regions are gold layer

upper side of tribofilm. For the thin tribofilm formed after 30 min, just one TEM image is shown, with FFT taken from the lower and upper sides of the tribofilm as indicated. One question that arises is whether the structures shown in Fig. 4 are representative. It should be noted that although small areas (50 nm width) of tribofilm are shown in Fig. 4, the FIB lamellae were ca $1 \mu \mathrm{m}$ wide and had the same structure across the whole width. Also, as will be indicated later in this paper, Test 6 was repeated in order to coat the tribofilm with $\mathrm{Cr}$ rather than $\mathrm{Au}$ prior to FIB. This showed a very similar nanocrystal structure to Fig. 3, indicating that the crystal structures shown are representative of the whole film and are repeatable.

In all of the lower-side regions, i.e. after 30-, 60- and 180-min rubbing, lattice fringes are observed; also FFT indicate that the tribofilms have periodic structure. These results show the presence of crystalline structures in the lower-side tribofilms. By contrast, the upper-side regions after 30- and 60-min rubbing do not show any lattice fringes, suggesting that the tribofilm in these regions consists of amorphous structures. However, after 180-min rubbing (Test 6), lattice fringes are observed in the upper-side tribofilm. Figure 5 is a close-up image of a region in the upper side of the tribofilm after 180-min rubbing extracted from Fig. 4 to more clearly show the nanocrystallinity. The tribofilm consists of packed crystalline domains of diameter ca. 3-5 $\mathrm{nm}$. The d-spacings (distances between planes of atoms giving rise to the diffraction peaks) were calculated from the FFTs to compare with known crystal structures. The main spacings found were $0.289,0.263$ and $0.168 \mathrm{~nm}$. This did not give a clear match to zinc ortho- or pyrophosphate although there was some similarity with zinc oxide. This suggests that the nanocrystals may have mixed composition. 


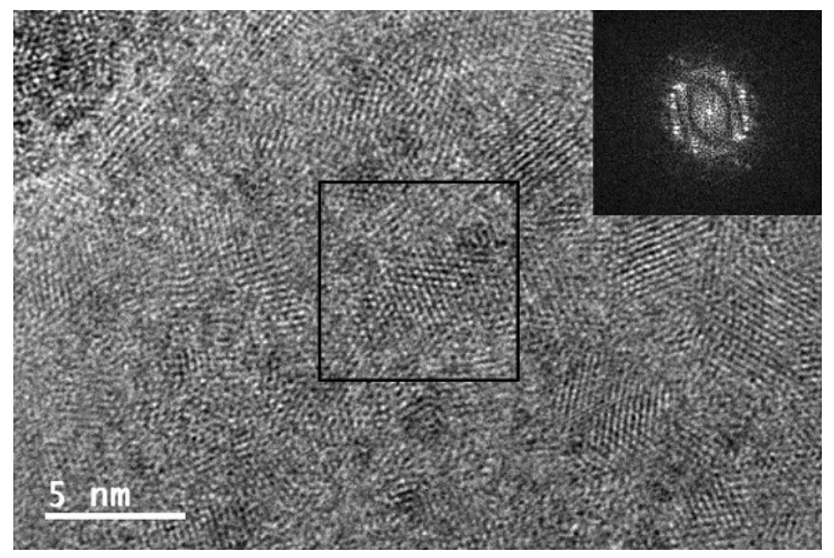

Fig. 5 Magnified view of crystalline region of ZDDP tribofilm in upper-side region (Test 6)

\subsubsection{Tribofilm Crystallinity After Rubbing in PAO}

Tribofilm crystallinity before and after the replacement of ZDDP2 solution by PAO was compared to investigate the effect of tribofilm crystallinity on durability. Figure 6 shows the cross-sectional TEM images of ZDDP tribofilm after 60-min rubbing in ZDDP2 (Test 3) and after 5 min of further rubbing in PAO (Test 4). The TEM image of the tribofilm after 60-min rubbing was obtained at low magnification (Test 3) to enable the entire tribofilm to be seen. FFTs of three regions, between 0 and $20 \mathrm{~nm}, 20$ and $40 \mathrm{~nm}$, and 40 and $70 \mathrm{~nm}$ from the steel substrate, are shown. While the crystalline structures are observed between 0 and $20 \mathrm{~nm}$ and 20 and $40 \mathrm{~nm}$ from the steel substrate, amorphous structures are confirmed in the uppermost $40-70 \mathrm{~nm}$ region. After 5-min rubbing in PAO, the TEM images show that 30 to $40 \mathrm{~nm}$ of tribofilm has been removed, which agrees with the SLIM measurement in Fig. 3. Now all of the remaining tribofilm has a crystalline structure, suggesting that the 30to 40-nm tribofilm removed consisted mainly of amorphous material.

\subsection{Tribofilm Chemical Structure}

Tribofilm on rubbed MTM discs was analysed using XPS to correlate the tribofilm crystallinity and chemical structure. Figure 7 shows the $\mathrm{O} 1 \mathrm{~s}$ XPS spectra of ZDDP tribofilm in Tests 1, 3, 4 and 6 . The ratios of bridging oxygen (BO) to non-bridging oxygen (NBO) were calculated from the $\mathrm{O} 1 \mathrm{~s}$ BO bond peak area $(532.6 \pm 0.5 \mathrm{eV}$ in the red line) and NBO peak area $(531.2 \pm 0.5 \mathrm{eV}$ in the blue line) $[4,5]$. A higher
Fig. 6 TEM images and FFTs of ZDDP tribofilm formed after 60-min rubbing in ZDDP2 solution (Test 3 ) and after additional 5-min rubbing in PAO (Test 4)

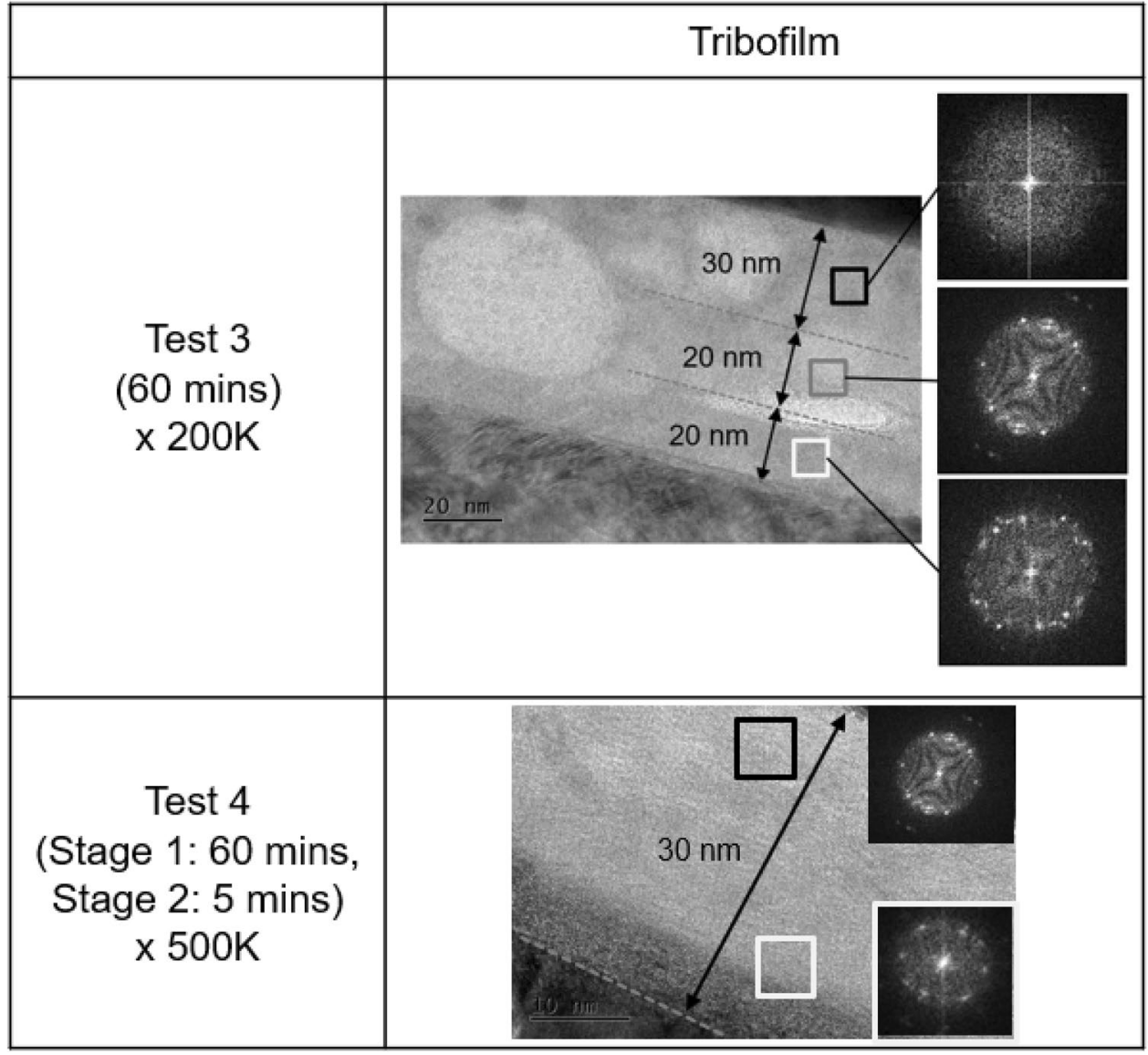




\begin{tabular}{|c|c|c|}
\hline & O 1s Spectra & $\mathrm{BO} / \mathrm{NBO}$ \\
\hline $\begin{array}{c}\text { Test } 1 \\
\text { (30 mins) }\end{array}$ & 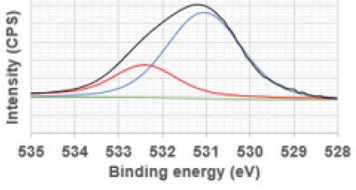 & 0.56 \\
\hline $\begin{array}{c}\text { Test } 3 \\
(60 \text { mins })\end{array}$ & $\begin{array}{lllll}535 & 534 & \begin{array}{l}533 \\
\text { Binding energy (eV) }\end{array} & 529 & 528\end{array}$ & 0.48 \\
\hline $\begin{array}{c}\text { Test } 4 \\
\text { (Stage 1: } 60 \text { mins, } \\
\text { Stage } 2: 5 \text { mins) }\end{array}$ & $\begin{array}{l}535534533532531530 \quad 529528 \\
\text { Binding energy (eV) }\end{array}$ & 0.31 \\
\hline $\begin{array}{c}\text { Test } 6 \\
\text { (180 mins) }\end{array}$ & 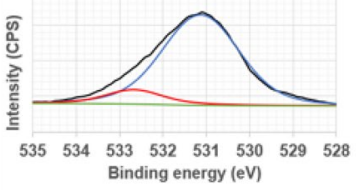 & 0.30 \\
\hline
\end{tabular}

Fig. $7 \mathrm{O}$ 1s XPS spectra of ZDDP tribofilm in different rubbing time with the $\mathrm{BO} / \mathrm{NBO}$ ratio (Tests 1, 3, 4 and 6)

$\mathrm{BO} / \mathrm{NBO}$ ratio indicates the longer polyphosphate chains, while a lower $\mathrm{BO} / \mathrm{NBO}$ ratio indicates shorter polyphosphate chains and orthophosphate. The BO/NBO ratio was $0.56,0.48$ and 0.30 after $30-, 60$ - and $180-$ min rubbing with ZDDP2, respectively (Tests 1, 3 and 6). These BO/NBO ratios suggest that zinc polyphosphate chains convert from longer chains to shorter chains during rubbing. It is important to note that the XPS signals originate almost entirely from within approximately $10 \mathrm{~nm}$ of the surface. In addition, the effect of rubbing in PAO subsequent to that in a ZDDP2 solution on tribofilm chemical structure was evaluated (Tests 3 and 4). After rubbing in $\mathrm{PAO}$ for $5 \mathrm{~min}$, the $\mathrm{BO} / \mathrm{NBO}$ ratio decreased from 0.48 to 0.31 , suggesting that longer-chain polyphosphates are removed after 5-min rubbing in PAO to expose shorter-chain polyphosphates.

\subsection{Influence of Temperature and ZDDP Alkyl Structure on Tribofilm Properties}

To understand the mechanism of polyphosphate crystallinity growth, the influencing factors on tribofilm growth and their effects on the evolution of tribofilm properties were evaluated at different temperatures and for different ZDDP alkyl structures.

\subsubsection{Tribofilm Durability}

Figure 8 shows the effect of rubbing temperature $\left(100{ }^{\circ} \mathrm{C}\right.$ and $60{ }^{\circ} \mathrm{C}$ ) on ZDDP tribofilm growth and durability. The ZDDP tribofilm grew more slowly at $60{ }^{\circ} \mathrm{C}$ than at $100{ }^{\circ} \mathrm{C}$ and stabilized at a lower thickness of ca. $60 \mathrm{~nm}$.

When the ZDDP2 solution was replaced by PAO after $180 \mathrm{~min}$ and rubbing continued, some of the ZDDP tribofilm formed at $60^{\circ} \mathrm{C}$ was removed (Test 10), unlike that formed at $100{ }^{\circ} \mathrm{C}$ (Test 8). These results show that ZDDP tribofilm becomes durable faster at higher temperature.

Figure 9 shows the effect of ZDDP alkyl structure on tribofilm durability. The tribotests using ZDDP1 were conducted up to 420 min to stabilize tribofilm growth. It is evident that ZDDP1 forms a tribofilm at a slower rate than ZDDP2 although the final tribofilm thickness is very similar. Although ZDDP2 tribofilm is not removed after $180 \mathrm{~min}$ when rubbed in PAO, $40 \mathrm{~nm}$ of ZDDP1 is removed after 180 min. In contrast, when ZDDP1 is replaced with PAO after $360 \mathrm{~min}$, tribofilm is not removed. These results show that tribofilm from ZDDP1 needs a longer rubbing time than ZDDP2 to become durable.

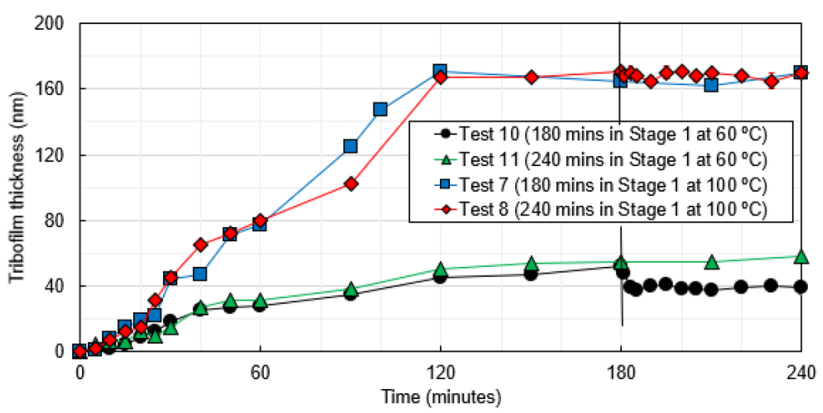

Fig. 8 ZDDP tribofilm evolution and its durability at $100{ }^{\circ} \mathrm{C}$ (Tests 7 and 8 ) and $60^{\circ} \mathrm{C}$ (Tests 10 and 11$)$

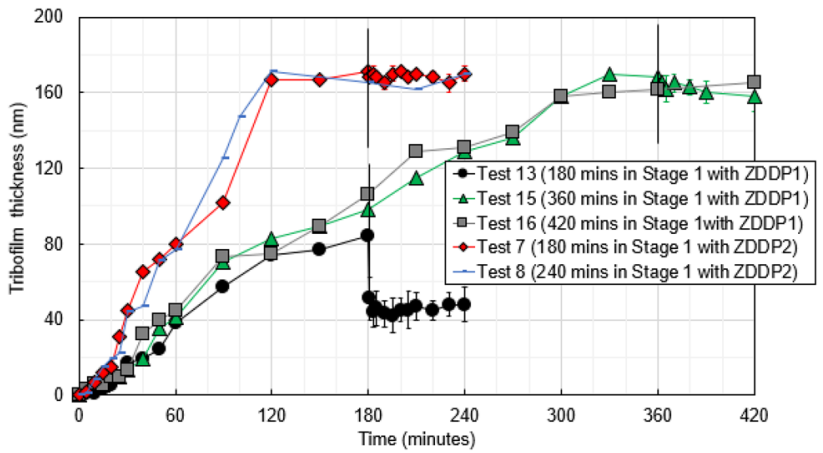

Fig. 9 Effect of ZDDP alkyl structure on tribofilm durability after different stage 1 rubbing times (ZDDP1: Tests 13, 15 and 16, ZDDP2: Tests 7 and 8 ) 


\subsubsection{Tribofilm Crystallinity}

Figure 10 shows the TEM images of ZDDP tribofilms with FFT after 180 -min rubbing at $60{ }^{\circ} \mathrm{C}$ and $100{ }^{\circ} \mathrm{C}$ test with ZDDP2 solution (Tests 6 and 9). TEM images of tribofilms after 180 -min and 360 -min rubbing at $100^{\circ} \mathrm{C}$ using ZDDP1 solution are also shown (Tests 12 and 14). For each tribofilm, the lower region close to the steel substrate is crystallinebased. However, ZDDP2 tribofilm after $180 \mathrm{~min}$ at $60{ }^{\circ} \mathrm{C}$ shows an amorphous layer in the upper regions, suggesting that crystallinity develops faster as rubbing temperature is increased. In the case of ZDDP1, amorphous layer formed in upper region after 180 -min rubbing at $100{ }^{\circ} \mathrm{C}$, but crystalline structures are then observed in upper region after $360 \mathrm{~min}$. These results suggest that ZDDP2 forms a crystalline structure faster than ZDDP1.

\subsubsection{Tribofilm Polyphosphate Structure}

Figure 11 shows the XPS O 1s spectra of ZDDP tribofilm after 180-min rubbing at $60{ }^{\circ} \mathrm{C}$ in ZDDP2 solution (Test 9) and at $100{ }^{\circ} \mathrm{C}$ in ZDDP1 solution after $180 \mathrm{~min}$ and $360 \mathrm{~min}$ (Tests 12 and 14). Spectra are compared with those from a tribofilm formed at $100{ }^{\circ} \mathrm{C}$ from ZDDP1 solution (Test 6). While the BO/NBO ratio after 180 -min rubbing in ZDDP2 at $100{ }^{\circ} \mathrm{C}$ is 0.30 , the $\mathrm{BO} / \mathrm{NBO}$ ratio is 0.57 at $60{ }^{\circ} \mathrm{C}$. This suggests that

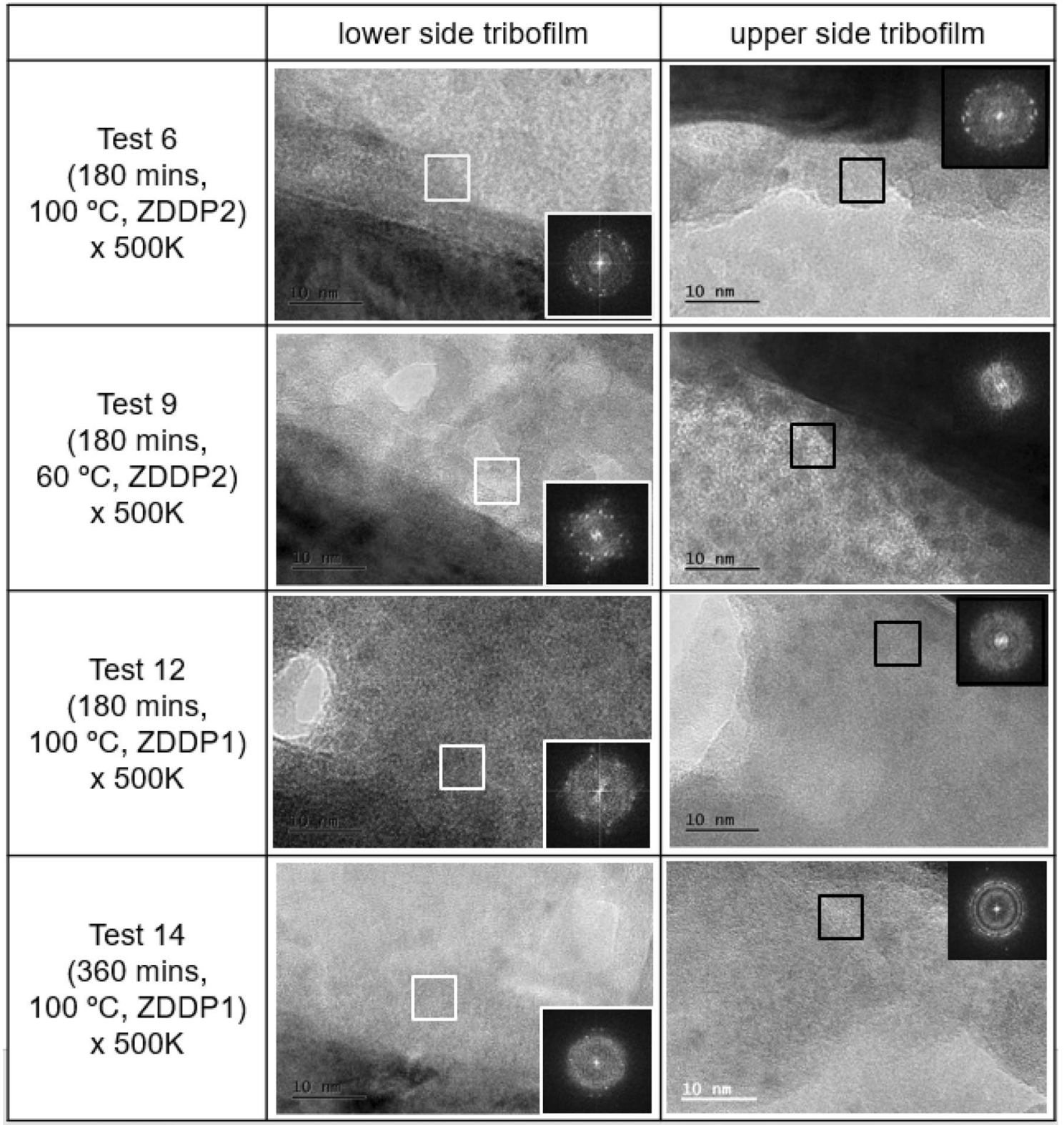

Fig. 10 TEM images and electron FFTs of ZDDP tribofilm at $60{ }^{\circ} \mathrm{C}$ and from primary ZDDP1 
Fig. 11 O 1s XPS spectra of ZDDP tribofilm at $60{ }^{\circ} \mathrm{C}$ from primary ZDDP1

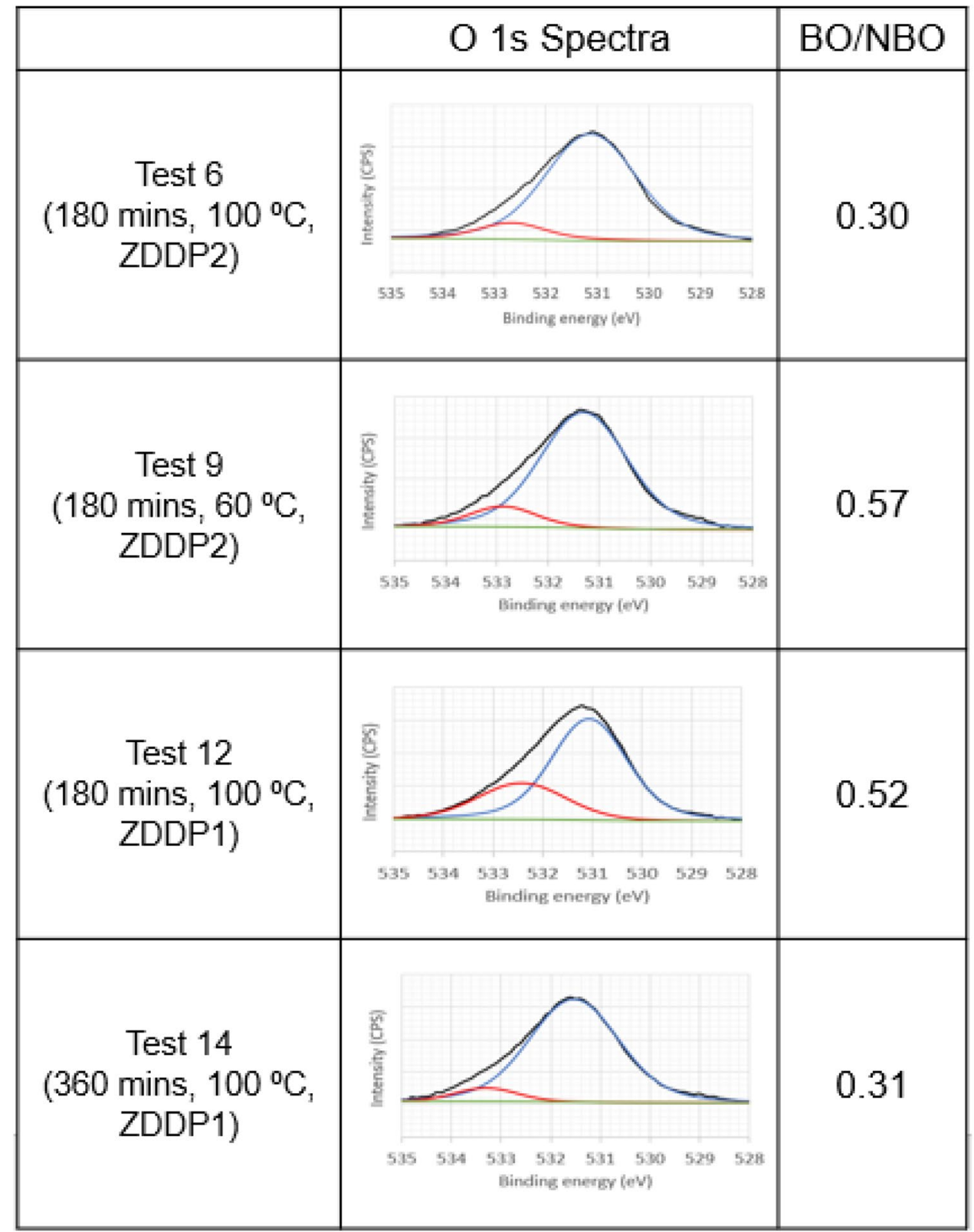

ZDDP2 tribofilms consist of longer polyphosphate chains after 180 -min rubbing at $60^{\circ} \mathrm{C}$ than at $100^{\circ} \mathrm{C}$. In the case of ZDDP1, polyphosphates are present after 180-min rubbing compared to ZDDP2, and tribofilm chain length then shortens after $360 \mathrm{~min}$, suggesting that zinc polyphosphate chains from ZDDP1 convert more slowly from longer chains to shorter chains with rubbing time than from ZDDP2.

\section{Discussion}

This study has provided clear evidence that a ZDDP tribofilm initially has an amorphous, glassy structure but that this converts to a nanocrystalline structure during prolonged rubbing. This conversion to nanocrystallinity begins in the tribofilm material adjacent to the ferrous substrate but later extends throughout the tribofilm. Tests in which an existing tribofilm is rubbed in base oil indicate that the amorphous tribofilm is relatively weak, but that once it converts to a nanocrystalline structure, it is highly durable and able to withstand extended rubbing in a ZDDP-free lubricant. Kalin et al. [15] measured the evolution of ZDDP tribofilm hardness with rubbing time using a nanoindenter. After 25-min rubbing, the hardness of ZDDP tribofilm was 2.4 GPa, whereas after 6-h rubbing the hardness it reached was $3.3 \mathrm{GPa}$, i.e. ZDDP tribofilm became harder with rubbing. Nanocrystalline structures are known to exhibit higher strength relative to amorphous or macro-crystalline ones 
since they combine lattice energy with effective obstruction of dislocation movement $[13,16,17]$. This evolution of ZDDP tribofilm properties during rubbing may help explain the finding that ZDDP tribofilms appear to adapt to applied stress by becoming stronger $[18,19]$.

The study also suggests that the evolution of ZDDP tribofilm to a durable, nanocrystalline structure results from a conversion of the molecular structure of the tribofilm from an initially formed polyphosphate to shorter chains and possibly primarily orthophosphate. In confirmation of previous studies, it finds that longer-chain polyphosphates in ZDDP tribofilms are converted to shorter-chain ones during extended rubbing $[8,20,21]$. A recent study of a ZDDP tribofilm using XANES has suggested that after a 2-h test at $100{ }^{\circ} \mathrm{C}$ the bulk of the tribofilm formed was almost entirely orthophosphate [22]. Crobu et al. [5] synthesized several types of zinc phosphate and found that zinc orthophosphate had a crystalline structure but that metaphosphate, polyphosphate and pyrophosphate were amorphous. When bulk metaphosphate and polyphosphate discs were rubbed against a steel ball counterface, the transferred material contained only short polyphosphates, while the discs also experienced a reduction in polyphosphate chain length. Berkani et al. [23] also studied the behaviour of meta- and orthophosphate in a tribocontact and found that at $120^{\circ} \mathrm{C}$ the metaphosphate was converted almost entirely to orthophosphate, while it became primarily pyrophosphate when rubbed against a steel counterface at room temperature.

The sequence of reactions that occurs during the formation of a ZDDP tribofilm is still not entirely understood, but after surface adsorption and initial formation of iron disulphide is believed to follow broadly the scheme indicated in Figs. 4 and 12 [8]. This involves initial transfer of alkyl groups from oxygen to sulphur, to convert the dialkyl thiophosphate to dithionyl phosphate. The phosphate is a strong nucleophile, while thionyl is an easy leaving group, so intermolecular reaction between thionyl phosphate species leads to rapid polymerization to form polyphosphate chains. These first two processes are accelerated by both temperature and stress and lead to formation of an amorphous polyphosphate tribofilm. The third stage, which is of particular interest in the current study, is subsequent depolymerization of this amorphous polyphosphate to primarily nanocrystalline orthophosphate.
Results from this study suggest that the depolymerization step is driven by prolonged rubbing at elevated temperature and thus by shear and heat. Berkani et al. [23] studied the properties of tribofilms formed by dispersed zinc metaphosphate powder and found that its depolymerization during rubbing was much less at room temperature than at $120^{\circ} \mathrm{C}$, while Crobu et al. [4] showed that the extent of depolymerization of meta- and polyphosphates was greater at higher load than lower load. It has been suggested that applied pressure alone may influence zinc phosphate chain length [24], but Berkani et al. [23] found no significant polymerization of orthophosphate or depolymerization of metaphosphate at high hydrostatic pressure. It is thus much more likely that asymmetric stresses such as shear and tensile stress, which increase proportionately with hydrostatic pressure in rubbing contacts, help drive depolymerization.

Along with heat and stress, depolymerization requires an oxygen-based nucleophile to break a $\mathrm{P}-\mathrm{O}-\mathrm{P}$ into two $\mathrm{P}-\mathrm{O}$ bonds and provision of a cation to balance the anionic charge generated. The nucleophile is likely to be water and/or oxide ions, while the cation might be $\mathrm{Zn}$ or Fe. Martin et al. [9] proposed that zinc polyphosphates reacted with iron(III) oxide in a hard acid/soft base reaction to form shorter-chain phosphates and $\mathrm{ZnO}$, while Heuberger et al. [8] also suggested that $\mathrm{Fe}$ ion from the steel substrate was the driving force to depolymerization. They found that while ZDDP thermal film was composed of longer polyphosphate, ZDDP tribofilm was composed of shorter polyphosphate, and XPS analysis showed larger amounts of Fe cations in tribofilm than thermal film, suggesting that $\mathrm{Fe}$ was released into the tribofilm during rubbing. Crobu et al. [5] rubbed a steel ball against a bulk zinc metaphosphate disc and found evidence of Fe transferred into the zinc phosphate, accompanied by reduction in the phosphate chain length. Berkani et al. [25] showed that addition of $\mathrm{FeOOH}$ to the lubricant promoted rapid depolymerization of tribofilms formed from zinc metaphosphate powder during rubbing. However, some depolymerization has also been observed in the absence of $\mathrm{Fe}$, in which case water and $\mathrm{Zn}$ cations are the most likely participants. Thus, Crobu et al. [5] found that some depolymerization of zinc metaphosphate and polyphosphate occurred when this was rubbed against a glass sphere, though much less than when rubbed against steel. These changes were ascribed to the action of adventitious water, and recently
Fig. 12 Suggested reaction sequence of tribofilm formation [8]

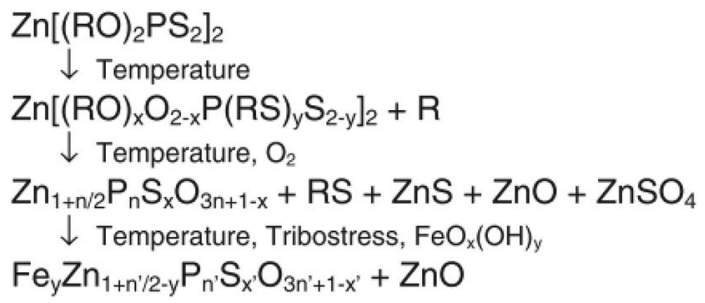

Starting compound: ZnDTP

Alkylation of sulphur

Polymerisation

Ion exchange and depolymerisation 
Parsaeian et al. [6] have shown that the deliberate addition of water does reduce the chain length of ZDDP tribofilms.

To investigate the effects of Fe cation distribution with rubbing time on depolymerization, an element distribution in cross-sectional tribofilm was analysed in the current study using EDX. While a protective Au coating was employed to protect tribofilm surface from Ga ion during the FIB preparation described in Sect. 3.3.1, a protective $\mathrm{Cr}$ coating was used prior to FIB for the EDX analysis. This is because EDX peaks of Au overlap with those of $\mathrm{Zn}$ and $\mathrm{P}$, but peaks of $\mathrm{Cr}$ do not, facilitating more precise analysis. Figure 13 shows the EDX line spectra and $\mathrm{Fe} / \mathrm{P}$ atomic ratio of tribofilm after Test 5 and Test 7 . It can be seen that, while Fe cation is only present in the lower and middle regions of the tribofilm after 60-min rubbing, Fe cation is also present in upper tribofilm after 180-min rubbing. These results showed that Fe cations from the steel substrate transfer to tribofilm with rubbing time. This transfer is most likely to be responsible, at least in part, for the depolymerization of polyphosphate and consequent development of crystallinity of the tribofilm. These results agree and support the ion exchange and depolymerization stage in Fig. 12.

The results for ZDDP1 solution show that the rate of tribofilm is slower for the primary ZDDP than for the secondary one tested, as generally reported in the literature [1]. However, the results also show that the conversion from amorphous to durable nanocrystalline structure is slower for primary than for secondary ZDDP.

The faster reaction rate of secondary ZDDPs is generally ascribed to the fact that the $\mathrm{C}-\mathrm{O}$ bonds in secondary ZDDPs are weaker than in primary ones, facilitating transfer of the alkyl to sulphur, together with the fact that the $\mathrm{P}-\mathrm{OR}$ and P-SR bonds in, or formed by, secondary ZDDPs are weaker due to the greater stability of the secondary OR and SR anions. This does not explain why the rate of depolymerization is slower for the primary ZDDP tribofilm, as evident in Fig. 8 since the depolymerization is driven by shear, heat, metal cations and water or oxide, which should be the same for both. It is possible that the primary ZDDP tribofilm retains more alkoxy or thionyl groups attached to the polyphosphate chains than the secondary ZDDP where these groups are lost more easily and that this retards depolymerization compared to the secondary ZDDP2 tribofilm.

The discovery that ZDDP tribofilms are initially amorphous and relatively weak and only become mechanically durable after depolymerization and crystallization has several important implications. One concerns research into ZDDP tribofilm kinetics. In the past, because the tribofilms tested after prolonged rubbing appeared to be very durable, it was assumed that there was negligible tribofilm removal during formation [1]. This allowed the rate of tribofilm thickness growth to be equated to the rate of ZDDP reaction, as the starting point for quantifying ZDDP tribofilm

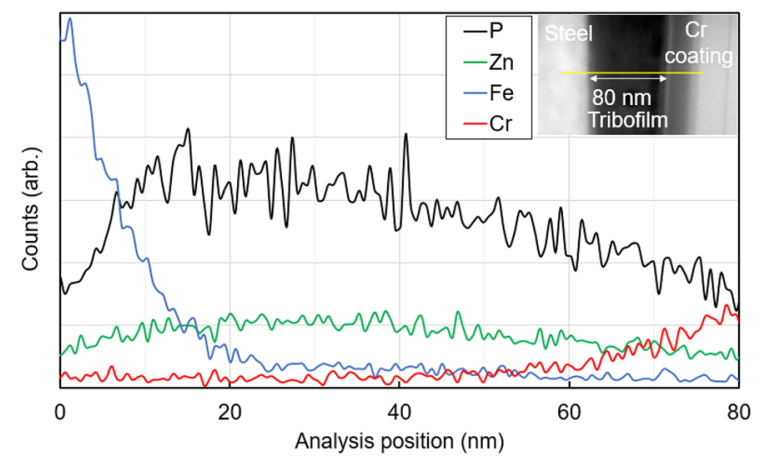

(a) EDX line profile of tribofilm in Test 5 (60 mins in Stage 1)

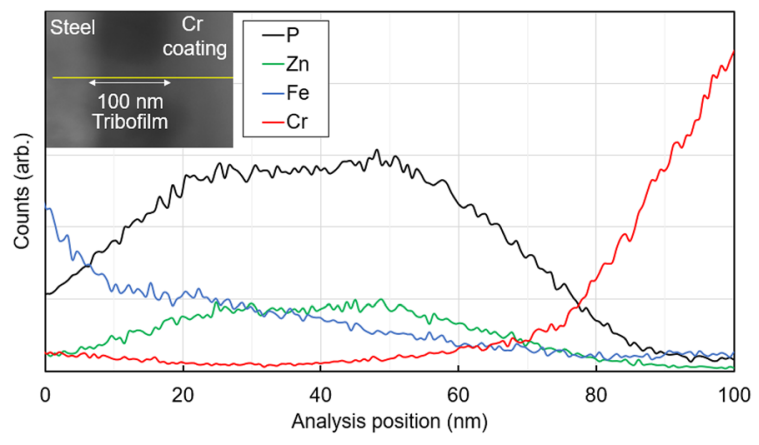

(b) EDX line profile of tribofilm in Test 7 (180 mins in Stage 1)

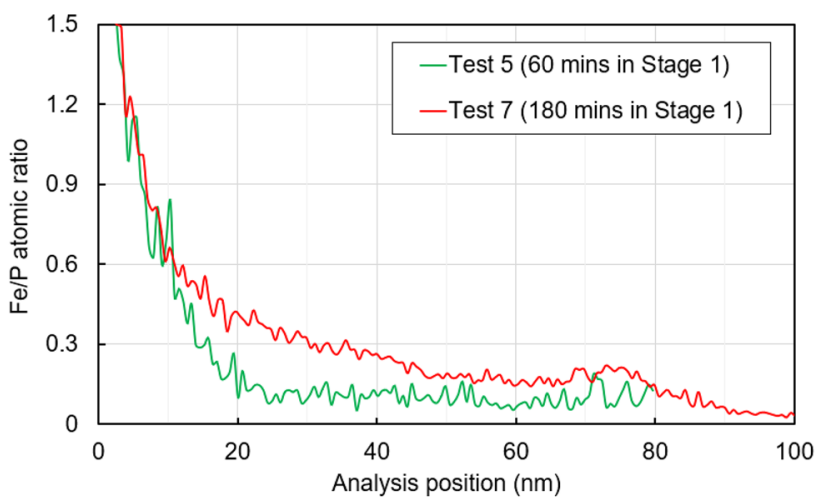

(c) $\mathrm{Fe} / \mathrm{P}$ ratio

Fig. 13 EDX line profiles and $\mathrm{Fe} / \mathrm{P}$ atomic ratio of the tribofilm in Tests 5 and 7. a EDX line profile of tribofilm in Test $5(60 \mathrm{~min}$ in stage 1). b EDX line profile of tribofilm in Test 7 (180 min in stage 1). $\mathbf{c ~ F e} / \mathrm{P}$ ratio

models [26]. However, it now appears that measured rates of ZDDP tribofilm growth may actually represent the difference between tribofilm formation and removal during the tribofilm development process. Development of useful kinetic models must thus find experimental ways to quantify each process separately.

The fact that tribofilm formation and removal processes both occur during ZDDP tribofilm growth also has practical implications since in some conditions tribofilm 
removal rate may exceed tribofilm formation rate. For example, ZDDP concentration should affect tribofilm formation rate but not removal rate, so at very low concentrations it may not be possible for protective ZDDP tribofilms to develop. High wear rates, in excess of those of the ZDDP-free base oil, have indeed been observed at very low ZDDP concentrations [27]. Although it appears that increase in contact pressure increases both tribofilm formation and removal rate [28], this is not likely to be the case at very high loads, where removal rate may well exceed formation rate; if this is the case there may be conditions in which ZDDP becomes no longer effective at reducing wear. Further work at high applied loads is needed to explore this. Finally, if, as seems very likely, Fe ions play an important role in tribofilm depolymerization and thus crystallization, it is likely that in the absence of such ions, tribofilm material might form but be very easily lost by rubbing. This may explain the apparent inability of ZDDP to form stable tribofilms on some DLCs. [29].

\section{Conclusions}

This study has shown using the MTM-SLIM tribometer and FIB-TEM that ZDDP tribofilms undergo a structural transformation during rubbing from a predominantly amorphous structure to one that is nanocrystalline. This occurs due to the depolymerization of initially formed polyphosphate chains to much shorter phosphates, possibly predominantly the orthophosphate, driven by temperature, applied stress and diffusion into the tribofilm of depolymerizing agents including iron cations. Importantly, this amorphous to nanocrystalline structural transformation results in the tribofilm becoming much stronger and more durable after extended rubbing. The results also show that primary ZDDP tribofilms convert from amorphous to nanocrystalline structure more slowly than those of secondary ZDDP.

The implications of this finding are considerable. It explains many previous observations such as the apparent strengthening of ZDDP tribofilms subjected to stress, the inability of ZDDP to form durable tribofilms on some nonmetallic materials and the impact of ZDDP concentration on antiwear effectiveness. The fact that initially formed amorphous ZDDP tribofilms are subject to both tribofilm formation and removal processes also provides strong pointers in the development of models of ZDDP tribofilms.

Acknowledgements The authors would like to thank Shell Lubricants Japan K.K. for supply of the base oils and additives for this study. Also, the authors would like to thank Dr. Katharina Marquardt from Imperial College London, Department of Materials, for useful discussions and information on analysis of the crystal structure of tribofilms.
Open Access This article is distributed under the terms of the Creative Commons Attribution 4.0 International License (http://creativecommons.org/licenses/by/4.0/), which permits unrestricted use, distribution, and reproduction in any medium, provided you give appropriate credit to the original author(s) and the source, provide a link to the Creative Commons license, and indicate if changes were made.

\section{References}

1. Fujita, H., Glovnea, R.P., Spikes, H.A.: Study of zinc dialkydithiophosphate antiwear film formation and removal processes, part I: experimental. Tribol. Trans. 48, 558-566 (2005)

2. Zhang, J., Yamaguchi, E., Spikes, H.A.: The antagonism between succinimide dispersants and a secondary zinc dialkyl dithiophosphate. Tribol. Trans. 57, 57-65 (2013)

3. Parsaeian, P., Ghanbarzadeh, A., Van Eijk, M.C., Nedelcu, I., Neville, A., Morina, A.: A new insight into the interfacial mechanisms of the tribofilm formed by zinc dialkyl dithiophosphate. Appl. Surf. Sci. 403, 472-486 (2017)

4. Crobu, M., Rossi, A., Mangolini, F., Spencer, N.D.: Chainlength-identification strategy in zinc polyphosphate glasses by means of XPS and ToF-SIMS. Anal. Bioanal. Chem. 403, 1415-1432 (2012)

5. Crobu, M., Rossi, A., Mangolini, F., Spencer, N.D.: Tribochemistry of bulk zinc metaphosphate glasses. Tribol. Lett. 39, 121-134 (2010)

6. Parsaeian, P., Van Eijk, M.C.P., Nedelcu, I., Neville, A., Morina, A.: Study of the interfacial mechanism of ZDDP tribofilm in humid environment and its effect on tribochemical wear; part I: experimental. Tribol. Int. 107, 135-143 (2017)

7. Dorgham, A., Azam, A., Morina, A., Neville, A.: On the transient decomposition and reaction kinetics of zinc dialkyldithiophosphate. ACS Appl. Mater. Interfaces. 10, 44803-44814 (2018)

8. Heuberger, R., Rossi, A., Spencer, N.D.: XPS study of the influence of temperature on $\mathrm{ZnDTP}$ tribofilm composition. Tribol. Lett. 25, 185-196 (2007)

9. Martin, J.M.: Antiwear mechanisms of zinc dithiophosphate: a chemical hardness approach. Tribol. Lett. 6, 1-8 (1999)

10. Yin, Z., Kasrai, M., Fuller, M., Bancroft, G.M., Fyfe, K., Tan, K.H.: Application of soft X-ray absorption spectroscopy in chemical characterization of antiwear films generated by ZDDP part I: the effects of physical parameters. Wear 202, 172-191 (1997)

11. Willermet, P.A., Dailey, D.P., Carter Iii, R.O., Schmitz, P.J., Zhu, W.: Mechanism of formation of antiwear films from zinc dialkyldithiophosphates. Tribol. Int. 28, 177-187 (1995)

12. Mosey, N.J., Müser, M.H., Woo, T.K.: Molecular mechanisms for the functionality of lubricant additives. Science 307, 1612 1615 (2005)

13. Serguee, A.V., Song, C., Valie, R.Z., Mukherjee, A.K.: Structure and properties of amorphous and nanocrystalline NiTi prepared by severe plastic deformation and annealing. Mater. Sci. Eng., A 339, 159-165 (2003)

14. Dawczyk, J., Ware, E., Ardakani, M., Russo, J., Spikes, H.A.: Use of FIB to study ZDDP tribofilms. Tribol. Lett. 66, 1-8 (2018)

15. Kalin, M., Oblak, E., Akbari, S.: Evolution of the nano-scale mechanical properties of tribofilms formed from low- and highSAPS oils and ZDDP on DLC coatings and steel. Tribol. Int. 96, 43-56 (2016)

16. Fan, C., Louzguine, D.V., Li, C., Inoue, A.: Nanocrystalline composites with high strength obtained in $\mathrm{Zr}-\mathrm{Ti}-\mathrm{Ni}-\mathrm{Cu}-\mathrm{Al}$ bulk amorphous alloys. Appl. Phys. Lett. 75, 340-342 (1999) 
17. Kalita, S.J., Bhardwaj, A., Bhatt, H.A.: Nanocrystalline calcium phosphate ceramics in biomedical engineering. Mater. Sci. Eng., C 27, 441-449 (2007)

18. Bell, J.C., Delargy, K.M., Seeney, A.M.: The removal of substrate material through Thick zinc dithiophosphate anti-wear films. Tribol. Ser. 21, 387-396 (1992)

19. Bec, S.A., Tonck, A., Georges, J.M., Coy, R.C., Bell, J.C., Roper, G.W.: Relationship between mechanical properties and structures of zinc dithiophosphate anti-wear films. Proc. R. Soc. A 455, 4181-4203 (1999)

20. Canning, G.W., Fuller, M.S., Bancroft, G.M., Kasrai, M., Cutler, J.N., De Stasio, G., Gilbert, B.: Spectromicroscopy of tribological films from engine oil additives. Part I. Films from ZDDP's. Tribol. Lett. 6, 159-169 (1999)

21. Martin, J.M., Onodera, T., De Barros Bouchet, M.I., Hatakeyama, N., Miyamoto, A.: Anti-wear chemistry of ZDDP and calcium borate nano-additive. Coupling experiments, chemical hardness predictions, and MD calculations. Tribol. Lett. 50, 95-104 (2013)

22. Dawczyk, J.U.: The effect of organic friction modifiers on ZDDP tribofilm. PhD Thesis, Imperial College London (2018)

23. Berkani, S., Dassenoy, F., Minfray, C., Martin, J.M., Cardon, H., Montagnac, G., Reynard, B.: Structural changes in tribo-stressed zinc polyphosphates. Tribol. Lett. 51, 489-498 (2013)
24. Nicholls, M.A., Do, T., Norton, P.R., Kasrai, M., Bancroft, G.M.: Review of the lubrication of metallic surfaces by zinc dialkyldithiophosphates. Tribol. Int. 38, 15-39 (2005)

25. Berkani, S., Dassenoy, F., Minfray, C., Belin, M., Vacher, B., Martin, J.M., Cardon, H., Montagnac, G., Reynard, B.: Model formation of ZDDP tribofilm from a mixture of zinc metaphosphate and goethite. Tribol. Int. 79, 197-203 (2014)

26. Ghanbarzadeh, A., Wilson, M., Morina, A., Dowson, D., Neville, A.: Development of a new mechano-chemical model in boundary lubrication. Tribol. Intern. 93, 573-582 (2016)

27. Fan, J., Spikes, H.A.: New test for mild lubricated wear in rollingsliding contacts. Tribol. Trans. ISSN 50, 145-153 (2007)

28. Zhang, J., Spikes, H.: On the mechanism of ZDDP antiwear film formation. Tribol. Lett. 63, 1-15 (2016)

29. Vengudusamy, B., Green, J.H., Lamb, G.D., Spikes, H.A.: Durability of ZDDP tribofilms formed in DLC/DLC contacts. Tribol. Lett. 51, 469-478 (2013)

Publisher's Note Springer Nature remains neutral with regard to jurisdictional claims in published maps and institutional affiliations. 